\title{
Polymer Chain Dynamics in a Random Environment: Heterogeneous Mobilities
}

\author{
K. Niedzwiedz, A. Wischnewski, M. Monkenbusch, and D. Richter \\ Institut für Festkörperforschung, Forschungszentrum Jülich, 52425 Jülich, Germany \\ A.-C. Genix, ${ }^{1}$ A. Arbe ${ }^{2}$ and J. Colmenero ${ }^{1,2}$ \\ ${ }^{1}$ Donostia International Physics Center, Paseo Manuel de Lardizabal 4, 20018 San Sebastian, Spain \\ ${ }^{2}$ Centro de Física de Materiales CSIC-UPV/EHU, Apartado 1072, 20080 San Sebastian, Spain
}

M. Strauch and E. Straube

Fachbereich Physik, Martin-Luther-Universität Halle-Wittenberg, D-06099 Halle, Germany

(Received 8 August 2006; published 17 April 2007)

\begin{abstract}
We present a neutron scattering investigation on a miscible blend of two polymers with greatly different glass-transition temperatures $T_{g}$. Under such conditions, the nearly frozen high- $T_{g}$ component imposes a random environment on the mobile chain. The results demand the consideration of a distribution of heterogeneous mobilities in the material and demonstrate that the larger scale dynamics of the fast component is not determined by the average local environment alone. This distribution of mobilities can be mapped quantitatively on the spectrum of local relaxation rates measured at high momentum transfers.

DOI: 10.1103/PhysRevLett.98.168301

PACS numbers: $83.80 . \mathrm{Tc}, 25.40 . \mathrm{Fq}$
\end{abstract}

The investigation of dynamic miscibility in polymer blends, i.e., the question of how friction arises in chemically heterogeneous systems at present is a very active area of research. At temperatures well above the component glass-transition temperatures $T_{g}$ the concept of segment self-concentration [1] provides a rather successful description of the component dynamics of a large number of polymer blends [2]. This self-concentration due to the chain connectivity is always enhanced locally and determines the component glass-transition behavior. On the other hand, in systems with greatly different component $T_{g}$ 's a decoupling of the dynamics of both components has been reported [poly(ethylene oxide)/poly(methyl methacrylate) (PEO/PMMA), polystyrene/poly(vinyl methyl ether), and PEO/poly(vinyl acetate)] [3-7]. In the system PEO/PMMA $\left(T_{g}^{\mathrm{PEO}} \approx 200 \mathrm{~K}, T_{g}^{\mathrm{PMMA}} \approx 400 \mathrm{~K}\right)$, recent NMR studies have found up to 12 orders of magnitude different local relaxation times [4]. In such a situation, the low- $T_{g}$ component moves in the random environment created by the frozen high- $T_{g}$ component - a qualitatively different scenario compared to that where both components move on similar time scales [8]. Beyond its interest for blend dynamics including plasticizing effects, this situation may create an experimental test bed facilitating investigations of polymer chains in random environments, which presently was mainly studied theoretically (see, e.g., $[9,10]$ and references therein).

This study is devoted to the space-time evolution of the PEO dynamics in PMMA at intermediate scales using quasielastic neutron scattering. We address the self- and the collective (single chain dynamic structure factor) dynamics. With incoherent scattering we have followed the mean square displacement (MSD) of the PEO segments in a time window up to about $1 \mathrm{~ns}$ and displacements up to
$1 \mathrm{~nm}$. While this local dynamics was found to be fast, the collective chain dynamics which could be followed up to about $80 \mathrm{~ns}$ by coherent scattering was apparently more than an order of magnitude slower. An appropriate scaling of the data did not show any characteristic length of confinement separating small and larger scale dynamics. Assuming a rather flat energy landscape but with locally varying mobility of the components, we introduce a distribution of friction coefficients and implement them in a Rouse model with random friction. In this way we were able to quantitatively and consistently describe the local, the intermediate, as well as the more global dynamics.

The deuterated PMMA (dPMMA) was purchased from Polymer Source Inc., Montreal, Canada. The polymers hPEO (protonated) and dPEO (99.8\% deuterated) were prepared by anionic polymerization using monomers purchased from CDN-isotopes (Quebec, Canada). The molar masses and polydispersity of the polymers were hPEO: $M_{n}=24 \mathrm{~kg} / \mathrm{mol}, M_{n} / M_{w}=1.03$; dPEO: $M_{n}=28.3 \mathrm{~kg} / \mathrm{mol}, M_{n} / M_{w}=1.07$; dPMMA: $M_{n}=$ $25.8 \mathrm{~kg} / \mathrm{mol}, M_{n} / M_{w}=1.04$.

For measuring the self-motion (incoherent scattering), hPEO/dPMMA samples with 25\%, 35\%, and 50\% hPEO, respectively, were prepared. To investigate the collective dynamics (single chain dynamic structure factor), samples with the above overall concentrations of PEO were cast, but they always contained $12.5 \%$ overall concentration of $\mathrm{hPEO}$ and the rest was deuterated.

The experiments were performed at the backscattering instrument BSS at the FZJ in Jülich (self motion up to $1 \mathrm{~ns}$ ), and at the neutron spin echo (NSE) spectrometers NSE at the FZJ in Jülich and IN15 at the ILL in Grenoble (collective chain motion up to $80 \mathrm{~ns}$ ). All these experiments were focused at small momentum transfer $(Q)$ val- 
ues $1 \mathrm{~nm}^{-1}<Q<6 \mathrm{~nm}^{-1}$. We investigated $T=350$, 375 , and $400 \mathrm{~K}$. In order to determine the coherent scattering contribution for all samples, polarized neutrons at the instrument DNS in Jülich were used. Finally the local picosecond dynamics of the $35 \% \mathrm{hPEO} / \mathrm{dPMMA}$ and the pure PEO samples were studied using the time of flight (TOF) spectrometer FOCUS at PSI in Villigen at large $Q$ 's $\left(Q \approx 20 \mathrm{~nm}^{-1}\right)$.

On intermediate length scales the dynamics of a polymer chain in the melt to a good approximation can be described in terms of the Rouse model [11]. This model treats the dynamics of a Gaussian chain in a heat bath which is experiencing entropic forces originating from the conformational chain entropy and viscous friction between the segments and their surroundings. At scales $Q R_{e} \gg 1$, where $R_{e}$ is the chain end-to-end distance, and the time scales investigated here, the intermediate self-dynamic structure factor has the Gaussian form

$$
S_{\text {self }}(Q, t)=\exp \left[-\frac{Q^{2}}{6}\left\langle r^{2}(t)\right\rangle\right]
$$

where $\left\langle r^{2}(t)\right\rangle=2\left(W \ell^{4} t / \pi\right)^{1 / 2}$ is the MSD; $W=$ $3 k_{B} T /\left(\zeta \ell^{2}\right)$ is the elementary Rouse rate. It is given by the ratio of the entropic force $3 k_{B} T / \ell^{2}$, with $\ell$ the segment length, and the friction coefficient $\zeta$. Also the single chain dynamic structure factor $S(Q, t)$ can be written as a function of the same scaling variable combining spatial and temporal scales. This scaling property is a consequence of the Rouse model involving no intrinsic length scale in the range of its validity. In the following the Rouse model with a certain averaged friction coefficient will be used as a first step to rationalize both the coherent as well as the incoherent scattering data.

In Gaussian approximation [Eq. (1)], the intermediate scattering function is directly related to the MSD of a segment. Figure 1 displays the MSDs obtained from an

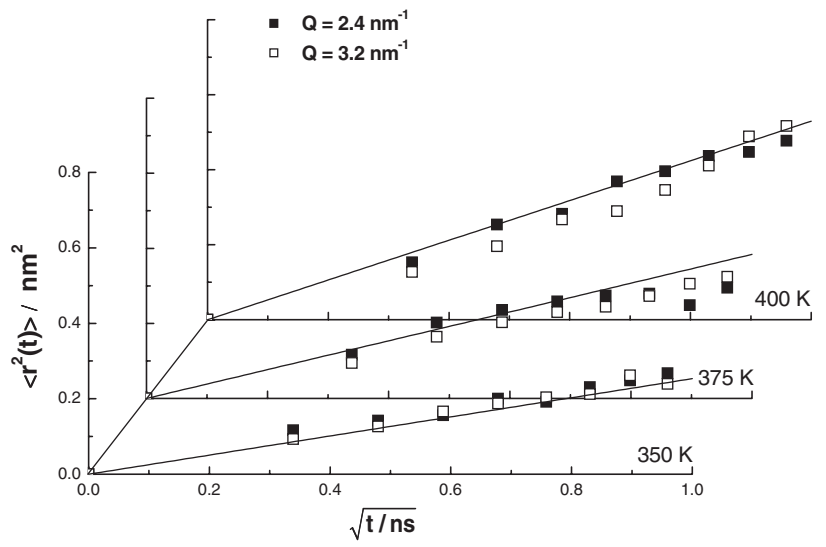

FIG. 1. Mean square displacements obtained from Fourier transformed backscattering spectra from the 35\% PEO/PMMA sample at different $Q$ and temperatures. The solid lines give the prediction of Eq. (1) using $W \ell^{4}$ values from a direct fit to the backscattering spectra. inversion of Eq. (1) applied to the Fourier transformed backscattering spectra from the 35\% PEO/PMMA sample. The figure includes data points obtained at the two smallest $Q$ values, $Q=2.4$ and $3.2 \mathrm{~nm}^{-1}$. In all cases the Fourier transformed data superimpose showing the validity of the Gaussian approach in the investigated $Q$ and time regime. In order to arrive at these results the original data needed to be corrected for the elastic coherent scattering originating from the contrast between the protonated PEO and the deuterated PMMA studied on DNS. In this way no ambiguity was introduced in the data. Plotting the obtained MSDs against $\sqrt{t}$, in all cases we obtain good agreement with the Rouse prediction. Depending on temperature, the maximum displacement of a PEO segment observed during about $1 \mathrm{~ns}$ amounts to $\sqrt{\left\langle r^{2}(t)\right\rangle}<1 \mathrm{~nm}$. The related values for the Rouse variable $W \ell^{4}$ are given in Table I, including the result for pure PEO.

So far we may state that the intermediate scale dynamics of PEO in a matrix of PMMA at temperatures where PMMA is nearly frozen-at $400 \mathrm{~K}$ for this system the PMMA local relaxation time is about $10 \mathrm{~ns}$ [5] compared to $6 \mathrm{ps}$ for PEO - is in good agreement with Rouse dynamics characterized by an average relaxation rate.

Figure 2 compares Fourier transformed spectra from pure PEO and 35\% PEO/PMMA obtained from the TOF instrument FOCUS at $T=400 \mathrm{~K}$. For the blend material Fourier transformed backscattering data are also included. At high $Q$, quasielastic neutron scattering observes local processes. Compared to the spectrum from pure PEO, the blend data are strongly broadened, demonstrating a broad distribution of local relaxation processes in the random PMMA environment. Both spectra merge at $S_{\text {self }}(Q, t=$ $0.4 \mathrm{ps})=0.4$ indicating the transition to microscopic dynamics which is not altered by blending. From the remaining decay we realize that a shift of the characteristic time from $1 \mathrm{ps}$ in pure PEO to about $6 \mathrm{ps}$ for PEO/PMMA is found-in excellent agreement with the NMR data [4] and also the ratio of the corresponding Rouse rates in Table I.

Figure 3 displays measurements of the single chain dynamic structure factor of PEO chains in the PMMA matrix. In order to achieve this result a deuterated PMMA matrix was used, where a mixture of deuterated and protonated PEO was immersed. In this context we note

TABLE I. Rouse rate as deduced from incoherent data and distribution width $\sigma$ for all samples, obtained from the analysis of the dynamic structure factor.

\begin{tabular}{ccc}
\hline \hline Sample & $W \ell^{4}\left(\mathrm{~nm}^{4} / \mathrm{ns}\right)$ & $\sigma$ \\
\hline Pure PEO $(T=400 \mathrm{~K})$ & 1.29 & $\ldots$ \\
25\% PEO/PMMA $(T=400 \mathrm{~K})$ & 0.1 & 2.1 \\
35\% PEO/PMMA $(T=400 \mathrm{~K})$ & 0.22 & 1.6 \\
35\% PEO/PMMA $(T=375 \mathrm{~K})$ & 0.12 & 1.8 \\
35\% PEO/PMMA $(T=350 \mathrm{~K})$ & 0.05 & 1.9 \\
50\% PEO/PMMA $(T=350 \mathrm{~K})$ & 0.13 & 1.6 \\
\hline \hline
\end{tabular}




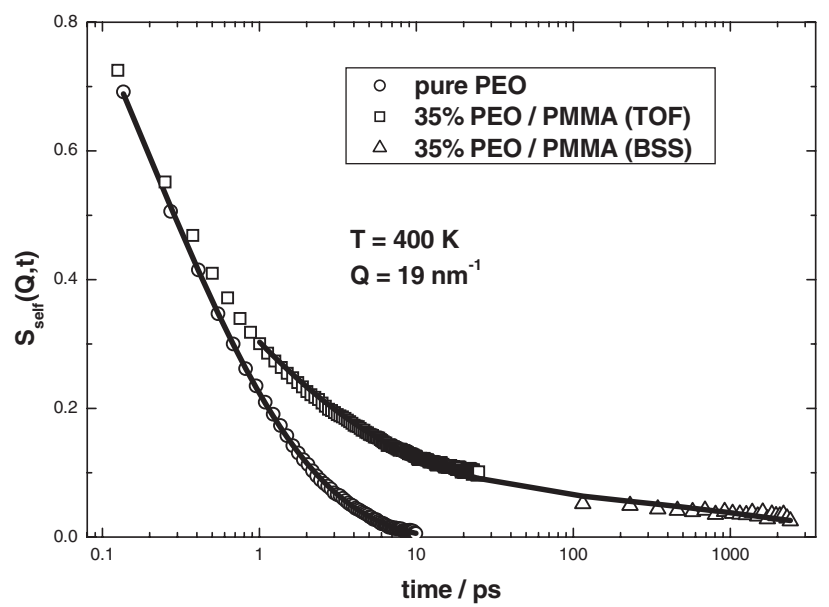

FIG. 2. Fourier transformed TOF data of pure PEO and $35 \%$ PEO/PMMA at $T=400 \mathrm{~K}$. For the blend system Fourier transformed backscattering data are also included. The solid line through the PEO data describes a fit with a stretched exponential $(\beta=0.5)$. Line through the blend data, see text.

that in the investigated $Q$ range and the proper time window pure PEO displays perfect Rouse dynamics [5]. The dotted lines in Fig. 3 are a result of a Rouse description based on the average Rouse relaxation rates obtained from the backscattering data at $1 \mathrm{~ns}$ scale. Obviously this description fails grossly predicting a by far too fast decay. Apparently the motion is strongly slowed down towards longer times or larger length scales.

We may roughly quantify this slowing down in fitting an effective smaller Rouse rate. This reveals retardations by factors of 4 to 20 (depending on PEO content and tem-

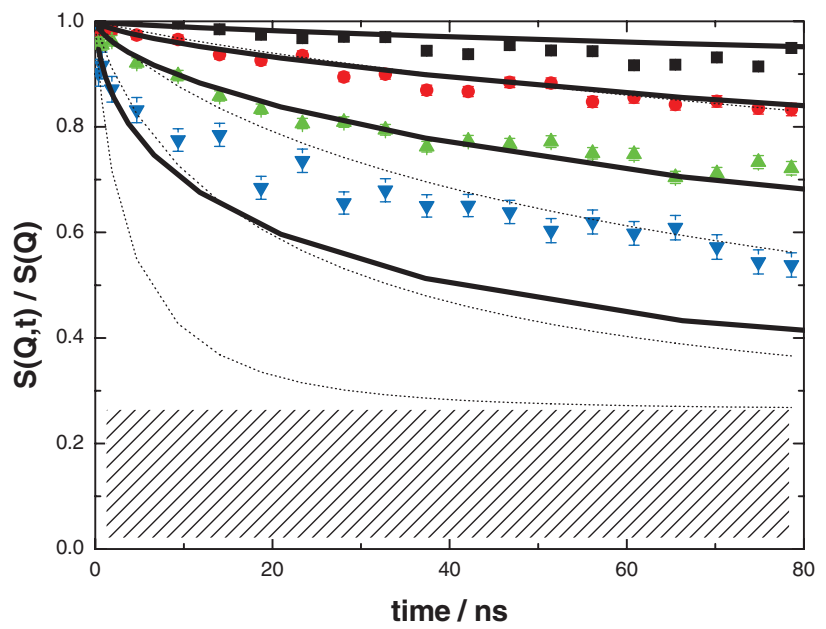

FIG. 3 (color). Single chain dynamic structure factor of the $35 \%$ PEO/PMMA system at $T=400 \mathrm{~K}$. $Q$ values are $1,1.5,2$, and $3 \mathrm{~nm}^{-1}$ from top to bottom. The dashed region represents the elastic contribution of 0.265 as calculated by dynamic RPA [16]. The dotted lines illustrate the Rouse theory with Rouse relaxation rate obtained from the backscattering data. The solid lines show the results of the Rouse model with random friction. perature) compared to the $1 \mathrm{~ns}$ scale. Recently forced Rayleigh scattering diffusion measurements of dilute short PEO chains (23 monomers) in a PMMA matrix [12] displayed a much stronger enhancement of the associated effective friction coefficient, $\zeta=10^{-2}$ dyns $/ \mathrm{cm}$ compared to the here observed value of $\zeta=3.2 \times 10^{-7}$ dyns $/ \mathrm{cm}$ measured at a scale comparable to the size of the small chains with NSE. The global dynamics of chain diffusion on a $\mu \mathrm{m}$ scale is apparently not related to the chain modes on a $80 \mathrm{~ns}$ time and about $1.5 \mathrm{~nm}$ spatial scale addressed with neutrons.

This very strong discrepancy might be taken as evidence for confinement, where locally fast motion could take place but globally the dynamics would be strongly reduced. We may ask whether the NSE data bear evidence for confinement effects with a characteristic confinement length. Figure 4 displays a presentation of the data of Fig. 3 but now as a function of the Rouse scaling variable combining both length and time scales. If there would be a characteristic length then the Rouse scaling, i.e., the merging of all $Q$ values to one master curve, would break down and different relaxation curves for different $Q$ vectors would emerge, as it is, e.g., the case for reptation [13] or chain dynamics in the presence of random obstacles [14]. Instead, all data superimpose to a common master curve with no sign of any characteristic length scale. This result bears evidence that the constraints on the PEO motion in the PMMA matrix are not dominantly random obstacles but rather random mobilities imposed by the PMMA.

In the following we show that a model of a Gaussian chain where the beads undergo random friction with a distribution of friction coefficients close to the distribution of relaxation times observed at high $Q$ is able to resolve most of the apparently contradictory experimental findings.

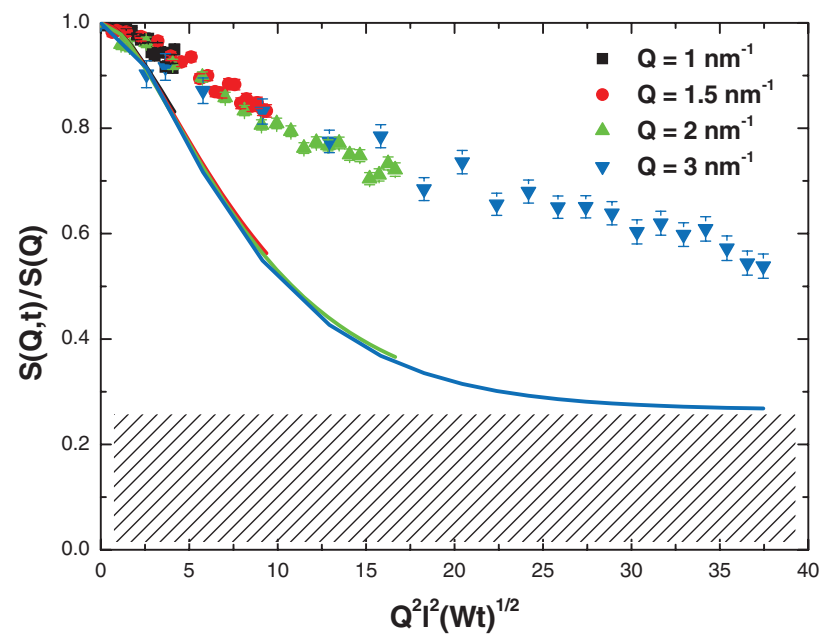

FIG. 4 (color). The same data as in Fig. 3 in the Rouse scaling representation. The dashed region is again the elastic contribution, the lines represent the Rouse theory with a friction factor as obtained from the backscattering experiment. 
In order to calculate the dynamic structure factor for such a chain we consider the Langevin equation for a polymer chain with $N$ beads (see, e.g., Chap. 1.5 in [13]). From the modified Langevin expression the equation of eigenvalues can be constructed by means of the usual normal mode ansatz. The calculation of the dynamic structure factor is known from the standard Rouse theory. The main ingredient of the calculation is a set of bead mobilities following a log-normal distribution centered around the average mobility obtained from the backscattering data, the only adjustable parameter being its width $\sigma$. The set of mobilities determines the dynamical matrix of the equation of eigenvalues which then is solved by standard numerical routines.

The structural averaging is realized by creating several chains as described above and computing the average of the structure factors. If the distribution function for the mobilities is known, all input parameters for the calculation of the dynamic structure factor are given by the blend properties (number and length of chain segments, elastic contribution depending on the blend composition). The solid lines in Fig. 3 show the best result which could be achieved for the 35\% PEO/PMMA sample at $T=400 \mathrm{~K}$ using $\sigma=1.6$. The three lowest $Q$ values are in very good agreement with the experimental data; however, the highest $Q$ data are reproduced at very short times only. The origin of this significant deviation at the highest $Q$ and longer times is still unclear, though it could be related to additional confinement effects imposed by the matrix. The values of $\sigma$ obtained by applying the same procedure to the other compositions and temperatures are given in Table I. As expected, the width of the distribution function increases with increasing PMMA concentration (in the limit of zero PMMA concentration it should go to zero) and within one blend $\sigma$ increases with decreasing temperature. We note that the program also reproduces the short time Rouse behavior with the observed average Rouse rate in a very consistent way.

Having in mind that at high $Q$ we observe directly the distribution of relaxation times, we now compare the results from the random Rouse model with the TOF spectra displayed in Fig. 2. We shift the average relaxation rate revealed by backscattering relative to pure PEO and take into account a small PMMA contribution revealed directly from high $Q$ backscattering spectra. Then we convolve the thus shifted PEO spectra with the log-normal distribution function using the result of $\sigma=1.6$ from the random Rouse model. With this we obtain the solid line in Fig. 2 in perfect agreement with the observed spectrum. Having in mind that $\sigma$ has been obtained from the NSE data and the shift of the average relaxation rate from the backscattering data, this agreement which combines data over 5 orders of magnitude in time is remarkable. Finally, diffusion is dominated by the lowest existing mobilities. Given the very broad distributions observed, the apparent discrepancy between diffusion and internal chain motion might relate to rare but important very low mobilities.

We presented a detailed analysis of quasielastic neutron scattering data from a PEO/PMMA blend. For short times the self-dynamics of PEO follows the Rouse expectation, however, with a significant larger friction coefficient compared to pure PEO. The collective chain dynamics at longer times is further retarded and shows no evidence for a characteristic length scale which would indicate confinement or random obstacles. Therefore we introduced a distribution of friction coefficients in the random Rouse model. With this we were able to quantitatively describe both the local as well as the dynamics at the chain level with a common set of parameters. The apparent strong disagreement of the dynamics at the chain level with that from macroscopic diffusion is not clear but might be related to the broad distribution of mobilities, to mesoscopic structure formation [15], or to entropic trapping [9], or to all of them.

The authors thank Dr. B. Farago (ILL), Dr. F. Juranyi (PSI), and Dr. W. Schweika (Jülich) for their help at the IN15, FOCUS, and DNS instruments, respectively, and Dr. J. Allgaier for the PEO synthesis.

[1] T. P. Lodge, and T.C.B. McLeish, Macromolecules 33, 5278 (2000).

[2] Y. He, T. R. Lutz, and M. D. Ediger, J. Chem. Phys. 119, 9956 (2003).

[3] C. Lorthioir, A. Alegría, and J. Colmenero, Phys. Rev. E 68, 031805 (2003).

[4] T. R. Lutz, Y. He, M. D. Ediger, H. Cao, G. Lin, and A. A. Jones, Macromolecules 36, 1724 (2003).

[5] A.-C. Genix, A. Arbe, F. Alvarez, J. Colmenero, L. Willner, and D. Richter, Phys. Rev. E 72, 031808 (2005).

[6] M. Tyagi, A. Arbe, J. Colmenero, B. Frick, and J. R. Stewart, Macromolecules 39, 3007 (2006).

[7] V. G. Sakai, C. Chen, J. K. Maranas, and Z. Chowdhuri, Macromolecules 37, 9975 (2004).

[8] S. Hoffmann, L. Willner, D. Richter, A. Arbe, J. Colmenero, and B. Farago, Phys. Rev. Lett. 85, 772 (2000).

[9] G. Migliorini, V. G. Rostiasvili, and T.A. Vilgis, Eur. Phys. J. B 33, 61 (2003).

[10] Statistics of Linear Polymers in Disordered Media, edited by B. Chakrabarti (Elsevier, Amsterdam, 2005).

[11] P. E. Rouse, J. Chem. Phys. 21, 1272 (1953).

[12] J. C. Haley and T. P. Lodge, J. Chem. Phys. 122, 234914 (2005).

[13] A. Wischnewski and D. Richter, Polymer Dynamics in Melts in "Polymer Melts and Mixtures," edited by G. Gompper and M. Schick (Wiley-VCH, Weinheim, 2006).

[14] P. G. de Gennes, J. Chem. Phys. 55, 572 (1971).

[15] X. Jin, S. Zhang, and J. Runt, Macromolecules 37, 8110 (2004).

[16] A. Z. Akcasu, Dynamic Light Scattering, The Method and Some Applications (Clarendon Press, Oxford, 1993). 